\title{
FACT - Highlights from more than Five Years of Unbiased Monitoring at TeV Energies
}

\author{
D. Dorner*1, J. Adam ${ }^{2}$, M.L. Ahnen ${ }^{3}$, D. Baack ${ }^{2}$, M. Balbo ${ }^{4}$, A. Biland ${ }^{3}$, M. Blank ${ }^{1}$, \\ T. Bretz $^{3}{ }^{a}$, K. Bruegge ${ }^{2}$, M. Bulinski ${ }^{2}$, J. Buss ${ }^{2}$, A. Dmytriiev ${ }^{4}$, S. Einecke ${ }^{2}$, \\ D. Elsaesser ${ }^{2}$, C. Hempfling ${ }^{1}$, T. Herbst ${ }^{1}$, D. Hildebrand ${ }^{3}$, L. Kortmann ${ }^{2}$, \\ L. Linhoff ${ }^{2}$, M. Mahlke ${ }^{3}{ }^{a}$, K. Mannheim ${ }^{1}$, S.A. Mueller ${ }^{3}$, D. Neise ${ }^{3}$, A. Neronov ${ }^{4}$, \\ M. Noethe ${ }^{2}$, J. Oberkirch ${ }^{2}$, A. Paravac ${ }^{1}$, F. Pauss ${ }^{3}$, W. Rhode ${ }^{2}$, B. Schleicher ${ }^{1}$, \\ F. Schulz ${ }^{2}$, A. Shukla ${ }^{1}$, V. Sliusar ${ }^{4}$, F. Temme ${ }^{2}$, J. Thaele ${ }^{2}$, R. Walter ${ }^{4}$ \\ ${ }^{1}$ Universität Würzburg, Institute for Theoretical Physics and Astrophysics Emil-Fischer-Str. 31, \\ 97074 Würzburg, Germany \\ ${ }^{2}$ TU Dortmund, Experimental Physics 5 Otto-Hahn-Str. 4, 44221 Dortmund, Germany \\ ${ }^{3}$ ETH Zurich, Institute for Particle Physics Otto-Stern-Weg 5, 8093 Zurich, Switzerland \\ ${ }^{4}$ University of Geneva, ISDC Data Center for Astrophysics, Chemin d'Ecogia 16, 1290 Versoix, \\ Switzerland \\ a also at RWTH Aachen University \\ E-mail: dornereastro.uni-wuerzburg.de
}

\begin{abstract}
The First G-APD Cherenkov Telescope (FACT) is monitoring blazars at TeV energies. Thanks to the observing strategy, the automatic operation and the usage of solid state photosensors (SiPM, aka G-APDs), the duty cycle of the instrument has been maximized and the observational gaps minimized. This provides a unprecedented, unbiased data sample of almost 9000 hours of data of which 2375 hours were taken in 2016.

An automatic quick look analysis provides results with low latency on a public website. More than 40 alerts have been sent in the last three years based on this.

To study the origin of the very high energy emission from blazars simultaneous multi-wavelength and multi-messenger observations are crucial to draw conclusions on the underlying emission mechanisms, e.g. to distinguish between leptonic and hadronic models.

FACT not only participates in multi-wavelength studies, correlation studies with other instruments and multi-messenger studies, but also collects time-resolved spectral energy distributions using a target-of-opportunity program with X-ray satellites.

At $\mathrm{TeV}$ energies, FACT provides an unprecedented, unbiased data sample. Using up to 1850 hours per source, the duty cycle of the sources and the characteristics of flares at $\mathrm{TeV}$ energies are studied.

In the presentation, the highlights from more than five years of monitoring will be summarized including several flaring activities of Mrk 421, Mrk 501 and 1ES 1959+650.
\end{abstract}

35th International Cosmic Ray Conference - ICRC2017

10-20 July, 2017

Bexco, Busan, Korea

${ }^{*}$ Speaker. 


\section{FACT - Ideal Monitoring Telescope at TeV Energies}

The First G-APD Cherenkov Telescope (FACT) is located at $2200 \mathrm{~m}$ a.s.l. in the Observatorio del Roque de los Muchachos, La Palma, Spain. The project's major goals are the proof-of-principle of silicon-based photosensors (SiPM, aka Geiger-Mode Avalanche Photo Diodes, G-APDs) in ground-based gamma-ray astronomy and long-term monitoring of blazars at $\mathrm{TeV}$ energies.

\subsection{SiPMs for Long-Term Monitoring}

The project is the first Imaging Air Cherenkov Telescope (IACT) to use SiPMs. Since its installation in October 2011, the camera has shown an outstanding performance nicely illustrating that SiPMs are an excellent alternative to photo multiplier tubes used so far in IACTs [1].

Long-term Stability The robustness and stable performance of SiPMs facilitates long-term monitoring: Using a feedback system, the gain of the SiPMs is kept stable in time and homogeneous over the camera [1] making a calibration redundant and facilitating the analysis of long-term data.

Observations During Bright Moon As SiPMs do not degrade when exposed to bright light, e.g. the full moon [2], the duty cycle of the telescope is enlarged. Not only the observation time per night is maximized, but also the observational gaps around full moon are minimized. This reduces the bias on variability studies like periodicity search [3] or calculating the duty cycle of the sources.

High Data Taking Efficiency The robustness of SiPMs fosters remote operation keeping the effort for maintenance low. Automatizing operation is also facilitated which in turn helps further maximizing the data taking efficiency as manual interaction is reduced to a minimum.

Thanks to the SiPMs, the data taking efficiency has been increased to up to $93 \%$ with respect to the time from start till end of astronomical twilight.With this increase in data taking efficiency, a total of more than 2375 hours of physics data have been taken in 2016.

\subsection{Blazars}

Active galactic nuclei (AGN) are extremely variable objects emitting radiation along the electromagnetic spectrum. Their spectral energy distribution (SED) features two peaks. For the subclass of blazars, the low-energy peak is in the optical to X-ray regime, while the high-energy peak is in gamma rays. In the unified scheme of AGN [4], blazars are classified as AGN with the jet pointed towards the observer. Thanks to particle acceleration in the jets and relativistic beaming, the emission is boosted to $\mathrm{TeV}$ energies, making blazars ideal targets for a small Cherenkov telescope like FACT.

Extreme Variability Blazars are known to exhibit extreme variability from time-scales of minutes [5] to years [6]. Due to the instruments' limited angular resolution, the origin of the gamma-ray emission from AGN is still unknown. Variability on very short time scales provides the possibility to constrain the size of the emission region [7]. While the variability on very short time scales has been investigated using observations of the duration of minutes to hours, the variability behaviour of blazars on time scales of hours to days is poorly known in the gamma-ray regime. To constrain the variability time scales and study the rise and decay times, continuous observations are needed. 
Multi-Wavelength and Multi-Messenger Aspects As blazars emit radiation along the electromagnetic spectrum, multi-wavelength (MWL) observations are crucial. While the low energy peak is attributed to synchrotron emission, the origin of the high energy emission is not yet understood. Diverse models predict different correlations in the MWL and also multi-messenger picture. While leptonic models assuming a single emission zone expect a correlation of the low and high energy peak, multi-zone models and hadronic models can accommodate more complex correlations allowing for time-lags and so-called orphan flares, i.e. only the high energy emission shows an outburst. As hadronic models expect neutrinos correlated with the gamma-ray emission, also multi-messenger studies are of large interest.

\subsection{FACT Monitoring Program}

To accommodate both MWL studies of variability and multi-messenger studies of blazars, the FACT Collaboration set up a monitoring program at $\mathrm{TeV}$ energies.

Continuous Long-Term Monitoring While larger IACTs like H.E.S.S., MAGIC and VERITAS have a manifold physics program, and therefore spend only short observations on each source, a low-cost instrument like FACT is ideal to do long exposures on a few selected sources. Such continuous observations with maximized duty cycle of the instrument and minimized observational gaps provide a unique opportunity to study the typical variability time scales of blazars and constrain the origin of the high-energy emission of blazars.

Source Sample Since more than five years, FACT is monitoring blazars at TeV energies. Apart from observing the Crab Nebula, the standard candle in $\mathrm{TeV}$ astronomy, for performance studies, the source sample and observation slots are optimized based on the performance of the telescope and the expected fluxes. Therefore the standard monitoring sample includes the brightest blazars: Mrk 421, Mrk 501 and 1ES 1959+650. In addition, 1ES 2344+51.4 has been monitored for many hours. Apart from that, also an exposure of more than 50 hours is available for $1 \mathrm{H} 0323+342$, PKS 0736+01 and V404 Cyg. A minor amount of time is used to follow-up alerts from other instruments.

Unbiased Observations While target-of-opportunity (ToO) observations are needed to study flares in detail, they bias the data sample towards higher fluxes. A crucial point in the FACT monitoring program is that the sources are observed regardless of their flux state, obtaining an unbiased data sample. Like this, FACT provides an unprecedented data sample to study the duty cycle of the sources and characterize their variability in gamma rays.

\section{Multi-Wavelength and Multi-Messenger Program}

To compile and study time-resolved blazar SEDs, simultaneous MWL observations are crucial.

\subsection{Flare Alerts}

To alert other instruments, a Quick Look Analysis (QLA) was set up providing results on a public website [8] with low latency. Details on the QLA can be found in [9] and [10]. 
Using the QLA results, alerts are sent to the community in case of interesting events. For Mrk 421 and Mrk 501, alerts are sent when the source gets brighter than $3 \mathrm{CU}$ while for other sources a flux limit of $0.5 \mathrm{CU}$ is applied requiring in addition a significance of at least $3 \sigma$ for the detection. For further alerts, the trigger criteria are adapted to the needs of the corresponding projects, e.g. triggering on fast rise or decay or with a different flux limit.

In total, the FACT Collaboration issued 44 flare alerts between March 2014 and June 2017: six alerts for Mrk 421 and Mrk 501 each, two alerts for 1ES 2344+51.4 and 30 alerts for 1ES 1959+650 which showed an exceptional flaring activity in summer 2016 [10].

As a follow-up of the FACT alerts, more sensitive instruments as H.E.S.S., MAGIC and VERITAS observed in several of these cases. Combined with FACT's dense monitoring, the high sensitivity observations provide extremely useful data samples. For example, the exceptional outburst of Mrk 501 in June 2012 is investigated together with MAGIC and VERITAS [11] and another bright outburst in June 2014 together with H.E.S.S. [12].

\subsection{Multi-Wavelength and Target-of-Opportunity Observations}

In 2015, FACT participated in a MWL campaign for the Seyfert galaxy 1 H0323+342. Since 2009, yearly MWL campaigns of Mrk 421 and Mrk 501 have been carried out with a cadence of roughly one week [13]. The denser FACT monitoring, including observations during bright moon, complements the data sample of the larger instruments providing access to additional time scales.

To enhance the MWL coverage and cadence during flares, a target-of-opportunity program has been set up based on the FACT blazar monitoring [10].

\subsection{Follow-Up Observations of MWL and Multi-Messenger Alerts}

Apart from sending alerts to trigger ToO observations, FACT also follows up MWL and multimessenger triggers on a best-effort basis. While priority is given to the monitoring program, the rest of the time is also dedicated to ToO observations provided that none of the more sensitive instruments is observing at the same time. In addition to few alerts from optical telescopes or the Fermi LAT instrument, alerts of neutrino events issued via the Astrophysical Multi-messenger Observatory Network (AMON, [14]) have been followed-up. In particular, the events HESE20160427 and HESE20160731 were observed as quickly as possible. From the position provided by IceCube no signal has been found. [put UL] More details on follow-up observations of neutrino alerts including data from other IACTs can be found in [15].

The combination of gamma-ray and neutrino data is also of interest to constrain the origin of astrophysical neutrinos and constrain the models predicting neutrinos from blazars. The FACT light curve of 1ES 1959+650 has been used to search for neutrinos [16].

\section{Results from More than Five Years of Monitoring}

Since more than five years, FACT is monitoring a small sample of sources. For Mrk 421 and Mrk 501, almost 2000 hours each have been collected. 1ES 2344+51.4 and 1ES 1959+650 have been monitored for more than 1000 hours each. Also the Crab Nebula has been observed for more than 1700 hours so far, including studies at large zenith distance and very bright moon light. In 
addition, few other sources have been observed for few to up to 550 hours, collecting data sample of almost 9000 hours in total between October 2011 and June 2017.

\subsection{ES 2344+51.4}

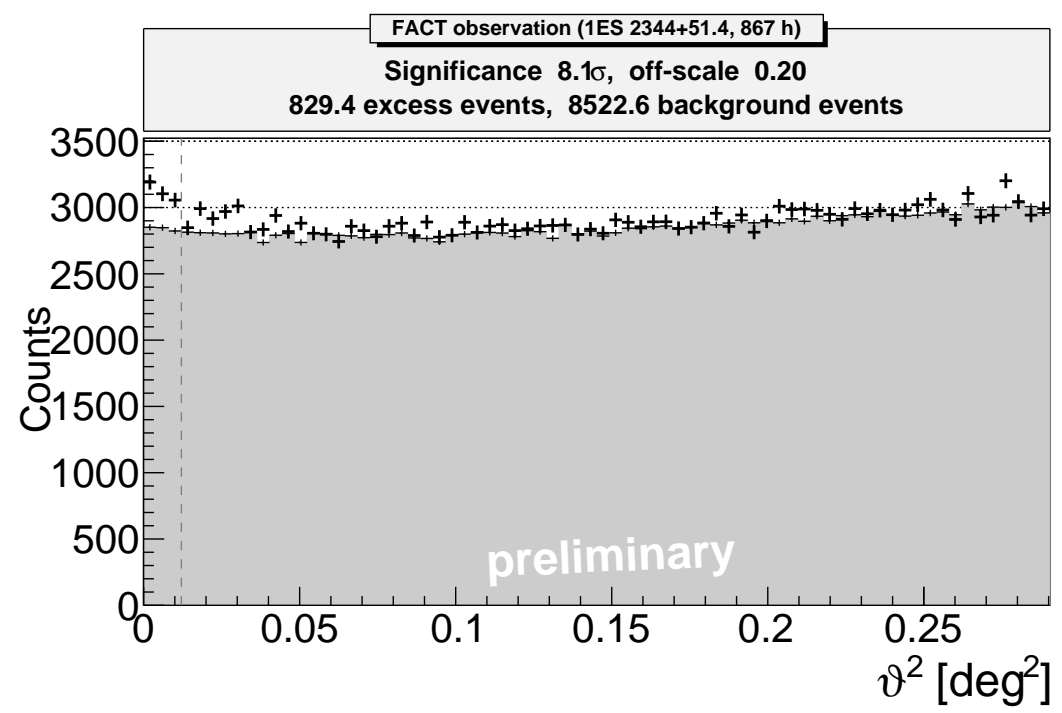

Figure 1: $\vartheta^{2}$-plot for the blazar 1ES 2344+51.4 for a total observation time of 867 hours.

While Mrk 421, Mrk 501 and 1ES 1959+650 showed bright outbursts with more than $3 \mathrm{CU}$ several times during the last years, the flux of 1ES 2344+51.4 did not increase above $0.3 \mathrm{CU}$ in the nightly binning. Despite of that, few periods of one to several weeks of enhanced activity were observed. From a total of 867 hours after data quality selection, 1ES $2344+51.4$ has been detected with $8.1 \sigma$ by FACT. Studying the long-term variability, a significant detection is found in four moon periods: Sep/Oct 2014, Nov/Dec 2015 and June to August 2016. In August 2016, MAGIC observations were triggered.

\subsection{Long-Term Blazar Light Curves}

Mrk 421 and Mrk 501 are known as the brightest TeV blazars on the Northern hemisphere. Both have been monitored with FACT since 2012.

Mrk 421 showed a bright outburst in April 2013 and some more flaring activity in 2015 and 2016 [17] as shown in Fig. 2 in the top panel. In December 2015, a flare of moderately high flux during the trigger window of the ToO program allowed to trigger INTEGRAL observations. In the following, also the Swift monitoring was denser, providing an interesting MWL data sample [10].

Mrk 501 showed bright flares in the first three years of monitoring, then the flux dropped to a generally lower level as shown in Fig. 2. The bright flare in June 2012 is studied together with MAGIC and VERITAS [11]. In 2014, six flare alerts have been sent to the community triggering also the H.E.S.S. observations of the bright flare in June 2014 [18]. The combined data set is being studied [12]. 

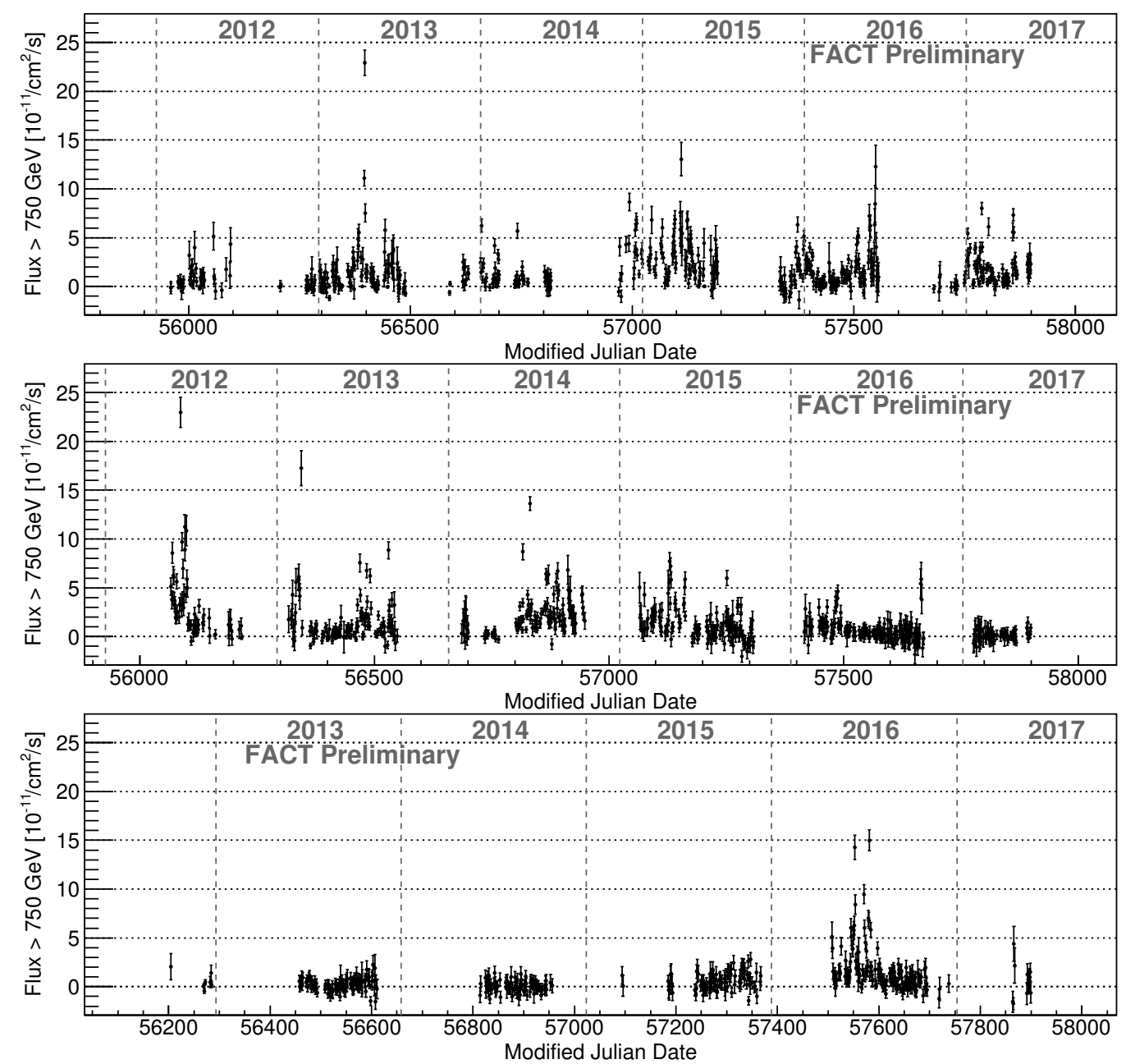

Figure 2: Light curves in nightly binning of the blazars Mrk 421 (top), Mrk 501 (middle) and 1ES 1959+650 (bottom) as measured by FACT.

1ES 1959+650 showed a low state in the first years of monitoring. In 2015, the flux increased and also X-ray activity was detected [19]. In 2016, an enhanced gamma-ray flux was found [20], and in the following the source showed two very bright flares [21, 22] and some more activity [23, 24]. The FACT light curve in 2016 is the densest daily light curve measured by an IACT so far and provides an ideal data sample to study the gamm-ray neutrino correlation [16].

Flux Distributions From the H.E.S.S. observations of the bright flare in June 2014, a hint for a preference of a lognormal behaviour of the fluxes during the flare night is found [25].

Studying the flux distributions for nightly fluxes (see Fig. 3), it is clear that they also do not follow a normal distribution.

\section{Acknowledgments}

The important contributions from ETH Zurich grants ETH-10.08-2 and ETH-27.12-1 as well as the funding by the Swiss SNF and the German BMBF (Verbundforschung Astro- und As- 

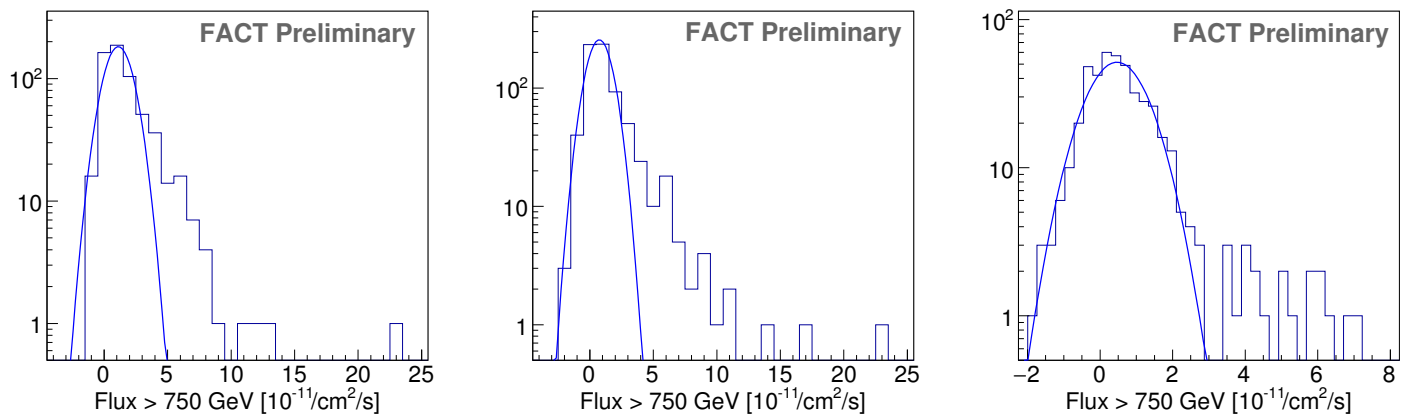

Figure 3: Flux distributions (black) of the blazars Mrk 421 (left), Mrk 501 (middle) and 1ES 1959+650 (right) as measured by FACT and fitted with a normal distribution (blue).

troteilchenphysik) and HAP (Helmoltz Alliance for Astroparticle Physics) are gratefully acknowledged. Part of this work is supported by Deutsche Forschungsgemeinschaft (DFG) within the Collaborative Research Center SFB 876 "Providing Information by Resource-Constrained Analysis", project C3. We are thankful for the very valuable contributions from E. Lorenz, D. Renker and G. Viertel during the early phase of the project. We thank the Instituto de Astrofisica de Canarias for allowing us to operate the telescope at the Observatorio del Roque de los Muchachos in La Palma, the Max-Planck-Institut für Physik for providing us with the mount of the former HEGRA CT3 telescope, and the MAGIC collaboration for their support.

\section{References}

[1] FACT collaboration, A. Biland, T. Bretz, J. Buß, V. Commichau, L. Djambazov, D. Dorner et al., Calibration and performance of the photon sensor response of FACT - the first G-APD Cherenkov telescope, Journal of Instrumentation 9 (Oct., 2014) P10012, [1403. 5747].

[2] FACT collaboration, M. L. Knoetig et al., FACT - Long-term stability and observations during strong Moon light, ArXiv e-prints (July, 2013) , [1307. 6116].

[3] FACT collaboration, M. Mahlke et al., FACT - Searching for periodicity in five-year light-curves of Active Galactic Nuclei, PoS ICRC2017 (2017) 612.

[4] C. M. Urry and P. Padovani, Unified Schemes for Radio-Loud Active Galactic Nuclei, PASP 107 (Sept., 1995) 803, [astro-ph/9506063].

[5] MAGIC collaboration, J. Albert et al., Variable Very High Energy $\gamma$-Ray Emission from Markarian 501, ApJ 669 (Nov., 2007) 862-883, [astro-ph/ 0702008 ].

[6] VERITAS collaboration, V. A. Acciari et al., Observation of Markarian 421 in TeV gamma rays over a 14-year time span, Astropart. Phys. 54 (2014) 1-10, [1310 . 8150].

[7] MAGIC collaboration, J. Aleksić et al., Black hole lightning due to particle acceleration at subhorizon scales, Science 346 (Nov., 2014) 1080-1084, [1412.4936].

[8] FACT collaboration, D. Dorner and et al., "FACT Quick-Look Analysis: http://www.fact-project.org/monitoring."

[9] D. Dorner, M. L. Ahnen, M. Bergmann, A. Biland, M. Balbo, T. Bretz et al., FACT - Monitoring Blazars at Very High Energies, ArXiv e-prints (Feb., 2015) , [1502. 02582]. 
[10] FACT collaboration, D. Dorner and et al., FACT - Time-Resolved Blazar SEDs, PoS ICRC2017 (2017) 608 .

[11] FACT, MAGIC, VERITAS collaboration, M. Ahnen and et al., The extreme HBL behavior of Mrk 501 during 2012, in prep. (2017) .

[12] FACT, H.E.S.S. collaboration, G. Cologna, N. Chakraborty, A. Jacholkowska, M. Lorentz, M. Mohamed, C. Perennes et al., The Exceptional Flare of Mrk 501 in 2014: Combined Observations with H.E.S.S. and FACT, ArXiv e-prints (Nov., 2016) , [1611.03983].

[13] MAGIC collaboration, J. Aleksić et al., The 2009 multiwavelength campaign on Mrk 421: Variability and correlation studies, A\&A 576 (Apr., 2015) A126, [1502.02650].

[14] M. W. E. Smith, D. B. Fox, D. F. Cowen, P. Mészáros, G. Tešić, J. Fixelle et al., The Astrophysical Multimessenger Observatory Network (AMON), Astroparticle Physics 45 (May, 2013) 56-70, [1211.5602].

[15] FACT, H.E.S.S., ICECUBE, MAGIC, VERITAS collaboration, M. Santander and et al., Searching for VHE gamma-ray emission associated with IceCube astrophysical neutrinos using FACT, H.E.S.S., MAGIC, and VERITAS, PoS ICRC2017 (2017) 618.

[16] FACT, ICECUBE, MAGIC collaboration, F. Kintscher and et al., IceCube Search for Neutrinos from 1ES 1959+650: Completing the Picture, PoS ICRC2017 (2017) 969.

[17] FACT AND HAWC collaboration, A. Biland et al., Enhanced and increasing activity in gamma rays and X-rays from the HBL Mrk421, ATel 9137 (2016) .

[18] H.E.S.S. collaboration, C. Stegmann, Increased VHE activity from Mrk 501 detected with H.E.S.S., The Astronomer's Telegram 6268 (June, 2014) .

[19] B. Kapanadze, D. Dorner, S. Vercellone, P. Romano, S. Kapanadze and T. Mdzinarishvili, A recent strong X-ray flaring activity of 1ES 1959+650 with possibly less efficient stochastic acceleration, MNRAS 461 (Sept., 2016) L26-L31.

[20] FACT, FERMi, MAGIC, VERITAS collaboration, S. Buson, J. D. Magill, D. Dorner, A. Biland, R. Mirzoyan and R. Mukherjee, Fermi-LAT, FACT, MAGIC and VERITAS detection of increasing gamma-ray activity from the high-energy peaked BL Lac object 1ES 1959+650, The Astronomer's Telegram 9010 (Apr., 2016) .

[21] FACT, FERMi, MAGIC, VERITAS collaboration, A. Biland, D. Dorner, R. Mirzoyan, R. Mukherjee, S. Buson and B. Kapanazde, Further increase of gamma-ray emission from the $H B L$ IES 1959+650, The Astronomer's Telegram 9148 (June, 2016) .

[22] FACT collaboration, A. Biland, FACT measures new maximum flux from the HBL 1ES 1959+650 at TeV energies, The Astronomer's Telegram 9239 (July, 2016) .

[23] FACT collaboration, A. Biland, FACT measures increased gamma-ray flux from the high-energy peaked BL Lac object 1ES 1959+650 since five nights, The Astronomer's Telegram 9139 (June, 2016)

[24] FACT, MAGIC collaboration, A. Biland and R. Mirzoyan, FACT and MAGIC measure an increased gamma-ray flux from the HBL 1ES 1959+650, The Astronomer's Telegram 9203 (July, 2016) .

[25] H.E.S.S. collaboration, N. Chakraborty, G. Cologna, M. A. Kastendieck, F. Rieger, C. Romoli, S. J. Wagner et al., Rapid variability at very high energies in Mrk 501, ArXiv e-prints (Sept., 2015), [1509.04893]. 